Rev. SINAPSIS, Edición № 9, Vol. 2, Diciembre 2016

\title{
Normas Internacionales de Información Financiera y la gestión Contable- Financiera: Realidades y perspectivas
}

Dewis Edwin Álvarez Pincay. Lcdo. ${ }^{(1)}$

Carlos Artemidoro Zea Barahona. Mg. ${ }^{(2)}$

Sara Geoconda Soledispa Reyes. Ing. ${ }^{(3)}$

Sandra Patricia Toala Bozada. Ing. ${ }^{(4)}$

$(1,2,3,4)$ Universidad Estatal del Sur de Manabí, Jipijapa, Ecuador

Contacto: $\underline{m \_d o 10 @ h o t m a i l . c o m ~}$

Receptado: 20/09/2016

Aceptado: 18/11/2016

\section{Resumen}

Las Normas Internacionales de Información Financiera (NIIF), han constituido una estandarización de la presentación de los estados financieros en un mundo globalizado, que incide de forma exponencial en el desarrollo de la comercialización, negocios e inversiones entre empresarios de muchos países. Las contribuciones relacionadas con las NIIF y su incidencia en la gestión contable-financiera estuvieron dirigidas a la comparabilidad, confiabilidad y la consistencia de la información contable, generalizados en el desarrollo de la presentación de los estados financieros para suministrar información acerca de la situación, desempeño y sostenimiento financiero de la empresa. Su objetivo fue concebir una metodología para la enseñanza problémica y una fácil práctica aplicación con procedimientos metodológicos generalizados, contribuyendo a elevar la relación que se produce entre la apropiación de la diversidad de contenidos y el carácter integrador de las diversas situaciones problémicas contables-financieras inherentes al desarrollo empresarial. El resultado estriba en aseverar la pertinencia de las NIIF sobre la base de las categorías de la enseñanza problémica profesional y su metodología desde el contexto globalizado en aras de lograr la interiorización del conocimiento y orientación valorativas que se reflejan gradualmente en los sentimientos, formas de pensar y comportamientos acordes a las exigencias socio-económicas. 
Rev. SINAPSIS, Edición № 9, Vol. 2, Diciembre 2016

ISSN 1390 - 9770

Palabras clave: Gestión empresarial, Enfoque Contable, Situaciones Financieras, Aplicaciones de normativas, Estados financieros.

\title{
International Financial Reporting Standards
}

\begin{abstract}
International Financial Reporting Standards (IFRS) have been a standardization of the presentation of financial statements in a globalized world, which has an exponential impact on the development of marketing, business and investments among entrepreneurs in many countries. Contributions related to IFRS and their impact on accounting-financial management were aimed at the comparability, reliability and consistency of accounting information, generalized in the development of the presentation of the financial statements to provide information about the situation, performance and financial sustainability of the company. Its objective was to devise a methodology for problem teaching and an easy practical application with generalized methodological procedures, contributing to raise the relationship between the appropriation of content diversity and the integrative nature of the various accountingfinancial problem situations inherent to Business development. The result is to assert the relevance of IFRSs based on the categories of professional problem teaching and its methodology from the globalized context in order to achieve the internalization of the knowledge and orientation values that are gradually reflected in the feelings, ways of thinking and behaviors according to socio-economic requirements.
\end{abstract}

Keyworks: Business management, Accounting approach, Financial situations, Regulatory applications, Financail estatements.

\section{Introducción}

Hace medio siglo, en la práctica profesional dentro del campo de la contabilidad, se ha testificado junto con muchos clientes internos y externo convergentes y divergentes en que es importante la estandarización de normas contables a nivel global.

También se observó el embargo petrolero, la conformación de la OPEP, la escalada del precio del petróleo y el brote financiero. Este gran fenómeno económico se estudió muy seriamente y esquematizado en forma técnica por expertos contables europeos. En cambio los profesionales estadounidenses lo desconocieron. 
Por un lado, los Principios de Contabilidad Generalmente Aceptados y por otro lado Las Normas Internacionales de Contabilidad, llevó a que cada ente que emitió estos principios y normas reportara en cada jurisdicción en base a normativa local, con un sesgo claramente fiscalista, y en base a procedimientos contables para efectos de consolidar los estados financieros y presentación a los accionistas (Hansen-Holm, 2009).

En efecto, todo esto llevó a que se generara una gran expectativa en este tema ya que tendría muchos impactos, los cuales han sido evaluados a medida que se han ido actualizando las normas.

En ese sentido, en el proceso de adopción, adaptación y convergencia a los International Financial Reporting Standars (IFRS), Estándares Internacionales de Información Financiera (EIIF), que fueron traducidas como Normas Internacionales de Información Financiera (NIIF), integran la información basada en las intenciones, intereses y necesidades de los usuarios Inversores, prestamistas y otros acreedores existentes y potenciales (Soto, 2015).

Lo anterior citado, lleva a comprender que el proceso de diseñar unas normas que aporten a usuarios internos y externos a tomar decisiones en base a una estandarización de presentación de los estados financieros.

En ese mismo sentido, los profesionales en contabilidad y auditoría han realizado un trabajo conjunto para la adopción de las NIIF lo que implica que las Normas Ecuatorianas de Contabilidad (NEC) ya no se apliquen, se debe en consideración que esto no fue proceso sencillo, pero el mismo ha salido avante ante una situación irreversible.

Todos los profesionales de la rama contable y de auditoría han estado actualizando sus conocimientos, mediante foros, seminarios, talleres, congresos, entre otros, tanto nacionales como internacionales, donde se aprovechó para discutir particularidades y 
Rev. SINAPSIS, Edición № 9, Vol. 2, Diciembre 2016

ISSN 1390 - 9770

peculiaridades que se consideraron para la implementación de las NIIF en empresas de distintos países.

Las Cuentas Consolidadas de las sociedades cotizadas en bolsas de la Unión Europea han comenzado a ser elaboradas, a partir de 2005, haciendo uso de las NIC/NIIF, por lo tanto las sociedades españolas cotizadas en nuestros mercados de valores han iniciado ya su rendición de cuentas aplicando dicha normativa. Pero más allá de este importante conjunto de empresas, entre las que se encuentran las de mayor tamaño y proyección pública, cabe señalar que la reforma contable en curso en España, está dando lugar a una profunda modificación del Plan General de Contabilidad, incorporando al mismo los nuevos criterios contables emanados de las NIC/NIIF, lo que supondrá en plazo inmediato la aplicación generalizada en la empresa española de la normativa contable internacional (Calvo, 2006)

Si bien es cierto, ahora en Ecuador ya las empresas presentan sus estados financieros bajo NIIF, pero aún se siguen generado dudas o temor por parte de los contadores cuando se les realiza una auditoría financiera, pues en ella se debe indicar si los mismos están conforme a las NIIF.

Es importante mencionar que la hipótesis planteada en esta investigación es "Las Normas Internacionales de Información Financiera, cumplen un rol protagónico a la hora de tomar decisiones económicas y financieras". En ese sentido el objetivo principal es, desarrollar habilidades y competencias en los inversionistas a la hora de tomar las decisiones económicas.

En esta investigación el principal componente son las NIIF y su aplicación para la toma de decisiones, en la cual se denota la relación existente entre la hipótesis y el diseño de la investigación.

A pesar de que es evidente el esfuerzo que hacen todos los involucrados en este tema, se presentan algunas complicaciones teóricas como por ejemplo, no se tiene mucha información de libros, artículos científicos, revistas indexadas y en lo relacionado a la 
Rev. SINAPSIS, Edición № 9, Vol. 2, Diciembre 2016

ISSN 1390 - 9770

práctica, aún hace falta conocer muchas más experiencias con empresas internacionales.

Atendiendo a la complejidad de los problemas actuales, los contadores enfrentan trascendentes desafíos, cuya solución exige el mejoramiento permanente de la profesionalización contable, financiera y de auditoría, ya que la fuerza más poderosa con la que contamos para dar respuesta a los retos globales, y formar la personalidad de los nuevos profesionales del siglo XXI, está en nuestra permanente auto preparación, capacitación y experiencia laboral. El establecimiento de las NIIF, acelera la evolución científica, tecnológica y organizacional de la economía actual, constituyéndose su estudio en una prioridad para lo que se deberá concebir la gestión y la innovación de forma sistemática, con la finalidad de cumplirlas de manera rápida y objetiva.

En ese orden de ideas, se puede manifestar que la información financiera se centra en las necesidades específicas de las personas externas a la empresa que toman decisiones, entre ellos los accionistas, los proveedores, los bancos y los organismos gubernamentales (Hongrem, 2000).

No hay duda de que la credibilidad actual de la información contable está en cuestión. La información contable tiene algunas deficiencias desde el punto de vista de los usuarios, tales como las diferencias contables internacionales, el precio de adquisición que a menudo está muy alejado del valor real de los activos, la contabilidad creativa y la no inclusión de la mayoría de los activos intangibles en las cuentas anuales (Amat, 2005).

Se vuelve una necesidad urgente el resolver el problema, de que en este campo no se está desarrollando de manera acelerada en Ecuador, si bien es cierto hay muchas expectativas por tratar de ganar experiencias y más conocimientos. Aún no somos los suficientemente peritos para ayudar a las empresas, y los accionistas para que tomen decisiones a nivel global, sino más bien en temas de cumplimiento de las normas para evitar sanciones. 
Rev. SINAPSIS, Edición № 9, Vol. 2, Diciembre 2016

ISSN 1390 - 9770

Los principales cambios que tiene la contabilidad denominada anteriormente PCGA Principios de Contabilidad Generalmente Aceptados cobijada en normas legales de un país de forma estática, cuando ahora que los cambios, principalmente por los avances tecnológicos y por la agilidad mental del hombre de negocios inagotable requiere adaptación de los registros contables hacia esos avances, lo cual lo ha entendido los organismos mundiales de regulación contable como la IASB (sigla en inglés) Junta de Normas Internacionales de Contabilidad o los USGAAP (sigla en inglés) o normas americanas (Gaitan, 2012).

Estos cambios a los cuales se hacen mención, es a lo que debemos estar preparados en todos los ámbitos pues implica una gran responsabilidad para la profesión del contador-auditor.

Cuando las políticas contables, según las NIIF, difieren de las que se aplicaron antes de la adopción de las NIIF, los ajustes resultantes se reconocen directamente en los resultados acumulados en la fecha de transición a las NIIF. Los estados financieros deben explicar cómo la transición de la contabilidad anterior a las NIIF afecta su situación financiera, su desempeño financiero y los flujos de efectivo de la empresa. Quien adopte por primera vez las NIIF debe desarrollar sus políticas contables para proporcionar información financiera relevante a los usuarios principales (inversionistas existentes y potenciales, prestamistas y otros acreedores) para utilizarla en la toma de decisiones acerca de la provisión de recursos a la entidad. Esa información debe representar fielmente, al término de su primer período de información según las NIIF, las transacciones que la entidad ha realizado (y otros eventos y condiciones a las que la entidad está sujeta), de conformidad con las NIIF. El desarrollo y la aplicación de esas políticas contables a menudo involucran el uso de juicio y la realización de estimados.

En ese sentido, los estados financieros incluyen información comparativa para uno o más períodos anteriores. Para mejorar la utilidad de la información financiera, cuando una entidad cambia una o más de sus políticas contables estos comparativos son generalmente modificados y presentados como si se hubiera aplicado siempre la nueva política contable. 
Rev. SINAPSIS, Edición № 9, Vol. 2, Diciembre 2016

ISSN 1390 - 9770

Por razones prácticas, las nuevas NIIF a veces incluyen disposiciones de transición que permiten que se aplique la nueva Norma, o partes específicas de la misma, de forma prospectiva (en lugar de hacerlo como si se hubiera aplicado siempre la nueva política contable) - ver la NIC 8 Políticas contables, cambios en las estimaciones contables y errores. Dichas disposiciones no se aplican a las entidades que adoptan las NIIF por primera vez.

En consecuencia, con el fin de proporcionar un punto de partida adecuado para la contabilidad de acuerdo a NIIF a un costo que no exceda sus beneficios, la NIIF 1 especifica algunas excepciones obligatorias y algunas exenciones opcionales a su principio general de que el que adopte por primera vez reconozca y mida todos los activos y pasivos en sus primeros estados financieros NIIF, como si siempre hubiera aplicado las NIIF. Por ejemplo, una entidad puede optar por no revisar la contabilidad de ninguna de las combinaciones de negocios que ocurrieron antes del inicio del período comparativo más antiguo que se presente. Alternativamente, puede elegir una fecha, antes del inicio del período comparativo más antiguo que se presente, y modificar todas las combinaciones de negocios que ocurran después de esa fecha. Otro ejemplo es que una entidad puede elegir medir un elemento de propiedad, planta o equipo con una revaluación anterior (si cumple con los criterios establecidos para hacerlo) o a su valor razonable, y utilizar esto como costo atribuido.

En los mercados de capital globalizados, las diferencias en la contabilidad hacen que los reportes financieros sean menos comprensibles y oscurecen las comparaciones que los inversionistas y los acreedores desean hacer. Los estándares de información financiera globales y de alta calidad, aplicados cuidadosamente y cumplidos rigurosamente, benefician a todos los proveedores de capital (Mantilla, 2013).

Ante lo citado se puede decir que, cuando la información financiera es de alta calidad, las decisiones que toman los dueños del negocio, futuros accionistas o inversores, son las correctas y que les satisfacen en todos los aspectos, pues genera una tranquilidad financiera ya que su inversión se encuentra segura. 
Rev. SINAPSIS, Edición № 9, Vol. 2, Diciembre 2016

ISSN 1390 - 9770

Es muy importante mencionar la consolidación de los estados financieros pues no se menciona mucho en lo relacionado a la presentación de la información financiera. La consolidación se refiere a grupos de empresas, es decir, estamos en un ámbito contable que va más allá de la empresa individual, para la que hemos visto en asignaturas precedentes cómo es su ciclo contable y cómo se elaboran sus cuentas anuales. Es un paso superior, que se refiere no a una empresa sino a un conjunto de ellas, pero no a un conjunto cualquiera de las sociedades sino a aquellos conjuntos que constituyen un grupo de empresas (Gutierrez, 2012).

La realidad muestra una manera diferente de hacer los negocios, cambios desde su concepción hasta su concreción, determinando la necesidad apremiante de revisar los modelos de negocio de las organizaciones sin que importen su tamaño y caracteristicas, con el fin de dar respuesta a las nuevas necesidades del mercado, donde la consideración de lo internacional no puede ignorarse, pues de hacerlo se corre el riesgo de ser desplazados por la competencia, que cada día está más presente con resultados y efectos inmediatos (Jiménez, 2015).

En efecto, tanto en la parte microeconómica y macroeconómica se vuelve casi una obligación revisar los modelos de negocios que manejan las organizaciones pues la competencia puede dejarlas en el camino causando hasta su desaparición, más aún cuando existe una crisis económica en la mayoría de los países muy acentuada.

Como se ha hecho mención anteriormente, una de las grandes diferencias que existen entre los estándares y prácticas de carácter local y sus similares de carácter internacional es que estos últimos se destacan por establecer tratamientos de índole aplicativa, en contraposición a los criterios conceptuales y filosóficos usados por las normativas locales. Es así como, en el momento del ejercicio profesional, la aplicación de los criterios orientados a la solución de problemas y situaciones de naturaleza práctica mejora considerablemente la efectividad de los procesos de análisis y verificación de la información contable (Figueroa, 2007).

Es evidente entonces, que al tener normativas locales y normativas internacionales, no hacen más que confundir y tomar decisiones erróneas a los futuros accionistas. Por eso 
destaca la importancia de estandarizar la información financiera-contable en aras de buscar una inversión segura, confiable y transparente.

Los estados financieros juegan un papel importante, ya que no solo proporcionan información relacionada con los resultados financieros sino que, además a través de estos estados se pueden obtener una serie de indicadores que permiten hacer un diagnóstico financiero para determinar cómo se comporta financieramente la empresa, cuál ha sido su crecimiento, cómo se encuentra en relación con las demás empresas del mismo sector, etc. (Roca, 2016).

Resulta oportuno manifestar, que los accionistas o potenciales dueños de negocios no sólo deben tener conocimiento sobre la información financiera, sino que además se debe tener pleno conocimiento o contar con una buena asesoría en lo relacionado a los indicadores financieros. Si bien es cierto, se cuenta con la información contablefinanciera pero falta lo más importante, saber interpretar las cifras y tomar las decisiones correctas.

Por todo lo anteriormente citado, se puede deducir que el contenido informativo es muy importante debido a que se explica claramente la problemática en lo relacionado a las NIIF y que el artículo propuesto se ajusta a la compresión acumulada de campo por cuanto se tiene una base bibliográfica sólida, técnica y profesional.

\section{Material y métodos}

El tipo de investigación documental y los métodos principales fueron el deductivo, inductivo y el histórico-lógico. Las técnicas de investigación fue la observación documental y revisión literaria, todo esto condujo a que se fundamentara el problema de manera profesional y técnica, generando un gran aporte al conocimiento en lo relacionado a las realidades y perspectivas de las NIIF, de la importancia de la información contable-financiera a la hora de tomar las decisiones en temas económicos.

Todo esto lleva una secuencia lógica que facilita el entendimiento y comprensión del artículo, iniciando una breve reseña histórica de las NIIF, su evolución, su adopción por primera vez, los impactos dentro de los esquemas contables, la importancia de 
Rev. SINAPSIS, Edición № 9, Vol. 2, Diciembre 2016

ISSN 1390 - 9770

contar con información relevante, consistente y confiable para que los futuros accionistas tomen la decisión de invertir en un negocio o empresa que esté generando rentabilidad y una gran porcentaje de ganancia en base a el dinero aportado cuyos riesgos financieros están siendo controlados y disminuidos.

\section{Resultados}

La aplicación de las NIIF ha dado lugar a un énfasis positivo y a una variabilidad elevada en cuanto a la concentración de un grupo de empresas de alto nivel que cotizan en la bolsa de valores sean estas locales o internacionales. Cuando se generan cambios circunstanciales y de políticas de estado se crea una incertidumbre a la hora de tomar las decisiones de los inversionistas. Actualmente todas las multinacionales presentan sus estados financieros bajo NIIF y que están a la vista y consideración de los futuros accionistas o a los dueños de los negocios. En este caso, la realidad de las empresas se ve reflejada al momento de presentar sus estados financieros y que casi el $100 \%$ de las empresas que cotizan en la bolsa de valores están siendo sujetas de seguimiento por parte de los inversionistas y por los entes de control. Este caso difiere de las PYMES ya que ellas tienen sus propias NIIF y que consta de 35 apartados.

En cuanto a la perspectiva de las empresas, se puede establecer que para seguir creciendo y que lleguen a ser consideradas como organizaciones modelos, necesitan de dinero fresco para aumentar su capital y para cumplir con los nuevos retos y desafíos que conlleva un mundo muy competitivo. Entonces se puede decir que el resultado para los futuros inversionistas y de la consecución de las metas y objetivos de las empresas, es muy halagador pues van a tomar sus decisiones en base a la información financiera basadas en las NIIF.

Para nadie es un secreto que el mundo está girando en torno a la globalización, y en el campo contable esta implica adoptar un cuerpo único de estándares de información financiera. De esta manera surge la preocupación a los Gerentes de la organización para con este proceso, e integrarse de la mejor manera.

Para la mayoría de los países es importante, unificar parámetros que permitan que los países que interviene en la economía, utilicen la misma estructura financiera y cambien 
Rev. SINAPSIS, Edición № 9, Vol. 2, Diciembre 2016

ISSN 1390 - 9770

los esquemas tradicionales que manejan, para de esta manera facilitar las negociaciones entre ellos bajo las mismas condiciones. El proceso de adoptar la llamada arquitectura financiera implica costos muy altos, lo que ha hecho más lento el proceso de estandarización.

Las Normas Internacionales de Información Financiera, se aplican a los estados financieros de cualquier empresa comercial, industrial o de negocios. La gerencia de la empresa puede formular estados financieros para su propio uso de varias y diferentes maneras, los cuales se adapten mejor para fines de administración interna.

\section{Discusión}

De acuerdo a los resultados obtenidos se evidencia que la hipótesis planteada se cumple pues los estados financieros presentados bajo NIIC inciden en la toma de decisiones de los accionistas y futuros inversionistas. Cabe recalcar que la información financiera que es consistente, confiable y comparable, presenta la realidad de las mismas y que genera una gran perspectiva en el sostenimiento y puesta en marcha del negocio.

Si bien es cierto, durante el trabajo de investigación se presentaron limitaciones tales como: poca información financiera de las empresas y poca información estadística, estas no afectaron a los resultados obtenidos, pues se investigó la evolución de las NIIF, su aplicación y adopción en las empresas.

Con relación a la información financiera que presentan las empresas, existe una seguridad razonable lo que genera valor agregado en la toma de decisiones y en el cumplimiento de las obligaciones legales. Esto disminuye el riesgo de que los inversionistas no quieran invertir en empresas sea del país que fuere, pues ellos confían en la información financiera que se le presentan. Puede que exista un temor cuando los estados financieros se presenten en moneda local y no en moneda dura como el dólar, en este caso se tiene que aplicar la convertibilidad para que de esta manera los valores sean revisados y valorados de manera estandarizada lo que facilita y agilita la toma de decisiones con una buena perspectiva. 
Rev. SINAPSIS, Edición № 9, Vol. 2, Diciembre 2016

ISSN 1390 - 9770

\section{Conclusión}

Para permitir la comparabilidad, la confiabilidad y la consistencia de la información contable se requiere en los diferentes países dentro de la llamada «globalización de la economía», la aplicación de reglas comunes para lograr el reconocimiento, la medición de los bienes y transacciones como para la revelación de información de los diferentes estados financieros, que exijan información comparable, transparente y de alta calidad, con el fin de ayudar a los participantes en los mercados de capitales de todo el mundo, y a otros usuarios, a tomar decisiones económicas correctas.

\section{Bibliografía}

Amat, O. (2005). Comprender la Normas Intrenacionales de información Financiera. NIIF 2006. España: Gestión 2000.

Calvo, L. C. (2006). Las Normas Internacionales de Información Financiera. Análisis y aplicación. Madrid-España: Thomson Civitas.

Figueroa, V. M. (2007). Aplicaciones Prácticas de las Normas Internacionales de Información Financiera. Costa Rica: Tecnológica de Costa Rica.

Gaitan, R. E. (2012). Estados Financieros Básicos bajo NIC/NIIF. Bogotá-Colombia: ECOE EDICIONES.

Gutierrez, S. M. (2012). Consolidación de los Estados Financieros. BarcelonaEspaña: Editorial UOC.

Hansen-Holm, M. A. (2009). Manual para implmentar las Normas Internacionales de Información Financiera. Guayaquil-Ecuador: Textos del Pacífico.

Hongrem, C. T. (2000). Introducción a la contabilidad financiera. M'xico: PEARSON.

Jiménez, J. M. (2015). Normas Internacionales de Información Financiera NIIF. Responsabilidad de la alta gerencia. Colombia: Externado.

Mantilla, S. A. (2013). Estándares/Normas NIIF. Bogotá-Colombia: Ecoe Ediciones.

Roca, C. M. (2016). Contabilidad Financiera para Contaduría y Administración. Bogotá-Colombia: Ecoe ediciones.

Soto, C. A. (2015). Plan de cuentas con NIIF. Taxonomía contable vs tesauro contable. Bogotá-Colombia: Ediciones de la U. 
Rev. SINAPSIS, Edición №9, Vol. 2, Diciembre 2016

ISSN 1390 - 9770

Financiera: Realidades y perspectivas www.itsup.edu.ec/myjournal 